\title{
CrimRxiv
}

\section{The Good Drone: How Social Movements Democratize Surveillance}

\section{Austin Choi-Fitzpatrick}

Published on: Jul 01, 2020

License: Creative Commons Attribution 4.0 International License (CC-BY 4.0). 
